\title{
Clinical Characteristics and Outcomes of Patients with Coronavirus Disease 2019-associated ARDS and the Early Intubation: A Two-Hospital Retrospective Cohort Study in Hubei, China
}

Jing-Lan Mu ( $\nabla$ mujinglanbb@gmail.com )

Nanjing Drum Tower Hospital: Nanjing University Medical School Affiliated Nanjing Drum Tower Hospital https://orcid.org/0000-0001-6548-5289

Ming-Zhe Qin

General Hospital of Central Theatre Command of PLA

Hui-Mei Sun

Southern Medical University Nanfang Hospital

\section{Bao-Sheng Guo}

Nanjing Drum Tower Hospital: Nanjing University Medical School Affiliated Nanjing Drum Tower Hospital

Shi-Da Qiu

Southern Medical University Nanfang Hospital

\section{Fu Zhang}

Southern Medical University Nanfang Hospital

\section{Huan Yang}

Southern Medical University Nanfang Hospital

Juan Nie

The People's Hospital of Honghu

\section{Xu-Dong Liu}

Sun Yat-sen University

\section{Wing-Fai Yeung}

The Hong Kong Polytechnic University

Xiao-Dong Liu

CUHK: The Chinese University of Hong Kong

\section{Bi-Xi Li}

General Hospital of Central Theatre Command of PLA

\section{Xiao-Yang Song}

General Hospital of Central Theatre Command of PLA

\section{Shao-Yan Lin}

Southern Medical University Nanfang Hospital 


\section{Ye-Hong Dong}

Southern Medical University Nanfang Hospital

\section{Ke-Xuan Liu}

Southern Medical University Nanfang Hospital

\section{Research}

Keywords: coronavirus disease 2019 , acute respiratory distress syndrome, early intubation, late intubation, in-hospital death

Posted Date: March 15th, 2021

DOl: https://doi.org/10.21203/rs.3.rs-307027/v1

License: (c) (i) This work is licensed under a Creative Commons Attribution 4.0 International License.

Read Full License 


\section{Abstract}

Background: More evidence in understanding the heterogeneity of COVID-19-associated acute respiratory distress syndrome (ARDS) and in improving strategy to increase the survival from the critical patients intubated is always needed. The study aimed to comprehensively explore the features of COVID-19associated ARDS and the features and outcomes between the early and late intubation groups.

Methods: This retrospective cohort included 65 adult COVID-19 inpatients with ARDS at two hospitals in Hubei, China. The ARDS in these patients was diagnosed according to the Berlin criteria. We defined intubation within 7 days of ARDS diagnosis as 'early' intubation and that performed from the eighth day as 'late' intubation based on literatures. The outcomes were invasive mechanical ventilation and inhospital death. The log-binomial regression models were used to explore the risk factors and the KaplanMeier statistic was used to estimate the risk of mortality.

Results: The median number of days from symptom onset to ARDS diagnosis was 11.0 (IQR, 8.0-13.0). Up to $84.1 \%$ COVID-19-related ARDS patients demonstrated multiple organ injuries. The mortality rates were $41.9 \%$ and $85.7 \%$ in moderate and severe ARDS. The early intubation and the late intubation had the differences in days from symptom onset/hospital admission/ARDS diagnosis to intubation $(P=0.023, P$ $=0.011, P<0.001)$. Compared with the early-intubation group, the late-intubation group showed less severity at admission (median oxygenation index 159.0 95\% Cl 134.0-203.0 vs. 133.9 95\% Cl 98.3-183.2), but required more aggressive therapies (ICU $80 \%$ vs. $70 \%$, CRRT $50 \%$ vs. $10 \%$, prone-position $50 \%$ vs. $30 \%$, and ECMO $50 \%$ vs. $10 \%$ ) and had higher risk to die at hospital (RR, 3.18; 95\% Cl 1.98-5.12).

Conclusion: The ARDS caused by COVID-19 was not typical ARDS due to prolonged onset time, multiple organ injuries, and higher mortalities. The late-intubation group showed less severity at admission but higher risk of in-hospital death than the early-intubation group.

\section{Key Points}

\section{Question}

Are features of ARDS caused by COVID-19 as similar as the previous ARDS? Is the late intubation at one week or later since ARDS diagnosis associated with the high mortality in COVID-19 patients compared to the early intubation?

\section{Findings}

In the retrospective cohort of 66 COVID-19 critical patients, we found that the ARDS was not the typically previous ARDS due to significantly prolonged onset time, multiple organ injuries, and higher mortalities. The late-intubation group showed less severity at admission but higher risk of in-hospital death compared with the early-intubation group.

\section{Meaning}


The findings had implications in modulating the management strategy for ARDS of COVID-19, and potentially in supporting the early or proactive intubation strategy.

\section{Introduction}

As of 27th February 2021, the coronavirus disease 2019 (COVID-19) pandemic has affected approximately 114 million people and caused over 2 million deaths worldwide [1]. The accumulated data suggest that $5-42 \%$ of COVID-19 patients develop severe illness that is characterized by acute respiratory distress syndrome (ARDS) [2, 3]. Our understanding about ARDS caused by COVID-19 remains remarkably different [4-6]. Some clinicians and researchers thought that the respiratory system mechanics of patients with ARDS, with or without COVID-19, were broadly similar [4], but others said that COVID-19-associated ARDS has distinctive features, including an primary vascular insult, that set it apart from previous ARDS and need to adjust the management strategies [5-7]. More evidence is always needed to clarify the features of ARDS to improve the treatment for the ongoing COVID-19 pandemic.

Another problem extremely required to be solved is the high mortality in COVID-19 patients who receive invasive mechanical ventilation (IMV) [8-10], which is the mainstay therapy for critical illness of COVID$19[11,12]$. A systematic review [13] analyzed 69 studies with 121,009 COVID-19 patients and reported that the case fatality rate (CFR) (mortality) of the patients receiving IMV was $45 \%$ (95\% $\mathrm{Cl} 39-52 \%)$. For the patients who completed their hospital stay, and the definite CFR with IMV across 11 studies from China and 21 studies from USA was $69 \%$ (95\% Cl 48-90\%) and 61\% (95\% Cl 50-72\%) respectively [13]. The mortality was even greater than $70 \%$ amongst patients aged above 60 years [13]. There are clinicians and researchers who think the timing of intubation is associated with the high mortality for COVID-19 patients $[14,15]$. The invasive mechanical ventilation thus should be used as proactive but not salvage therapy, to reduce the deaths $[7,16]$. There is a report that proposed the existence of two primary phenotypes of type $L$ (low lung compliance) and type $\mathrm{H}$ (high lung compliance) of COVID-19-associated ARDS $[7,17]$. Authors therefore advocated to perform proactive or early intubation in patients before excessive inspiratory efforts without worrying ventilator-induced lung injury as the high compliance results in tolerable strain [17]. Direct evidence on the consequences of delayed intubation in COVID-19 patients is not yet available. Early intubated patients seem to have better outcomes through personal experience. Analyses on retrospective data on this matter are urgently needed. In the studies published, there were a number of patients receiving intubation at one week or later after hospital admission compared with those receiving the therapy within one week $[15,18]$. We therefore wonder if such comparably late intubation is associated with the high mortality.

We performed a two-centre retrospective cohort to extensively investigate the characteristics of COVID-19associated ARDS patients, to explore the features and outcomes between the early and late intubation groups according to 1-week cutting point since ARDS diagnosis, and to study the predictors for the early intubation.

\section{Materials And Methods}




\section{Ethical approval}

This manuscript adheres to the applicable CONSORT guidelines. The study was approved by the Chinese Ethics Committee of Registering Clinical Trials (ChiECRCT20200113), and the requirement for informed consent was waived because of the retrospective study design and given the context of ongoing infectious diseases.

\section{Study design}

This retrospective cohort study included adult COVID-19 inpatients with ARDS ( $\geq 18$ years old) from the General Hospital of Central Theatre Command of PLA in Wuhan (a tertiary hospital) and from the People's Hospital of Honghu (a community hospital) in Jingzhou, a city close to Wuhan, in Hubei province, China. COVID-19 was diagnosed based on the World Health Organization (WHO)'s technical guidance on COVID-19, including confirmation with real-time reverse transcription polymerase chain reaction (RT-PCR) [19].

\section{Inclusion and exclusion criteria}

We identified patients with COVID-19-related ARDS admitted to the two hospitals from 26 January 2020 to 1 April 2020. The ARDS was diagnosed according to the Berlin criteria: development of acute, bilateral pulmonary infiltrates (determined by chest imaging) and hypoxemia $\left(\mathrm{PaO}_{2} / \mathrm{FIO}_{2} \leq 300 \mathrm{mmHg}\right.$ or $\mathrm{SpO}_{2} / \mathrm{FiO}_{2} \leq 315$ as a surrogate if no $\mathrm{PaO}_{2}$ data are available in the study) not primarily due to heart failure or volume overload [20]. Patients with COVID-19-related ARDS were included in the cohort independent of their positive end-expiratory pressure (PEEP) requirements. Therefore, patients receiving oxygen via a nasal cannula and facemask were included if they met the criteria of ARDS, in order to recognize more potential inpatients who later presented with hypoxemia respiratory failure warranting IMV. Patients were screened and included by reviewing their electronic medical records and laboratory results. We excluded COVID-19 patients who did not meet the ARDS diagnosis criteria, who did not have laboratory confirmation of ARDS, or had ARDS caused by other pathogens, such as bacteria, fungi, mycoplasma or chlamydia.

\section{Data collection}

We reviewed the electronic medical records, laboratory results and radiographic reports of all admitted COVID-19 patients. Using a modified version of the standardized case report form developed by the International Severe Acute Respiratory and Emerging Infection Consortium and WHO [21], we extracted data on the demographic characteristics, medical history, comorbidities, illness onset date, initial symptoms, vital signs on admission and other data such as the severity of pneumonia graded by physicians. We also extracted the results of routine hematological, biochemical and inflammation-related 
indices obtained within 72 hours of hospital admission. Further, we collected information on ARDS diagnosis, including chest imaging; $\mathrm{FiO}_{2}, \mathrm{PaO}_{2}$ or $\mathrm{SpO}_{2}$ values to calculate oxygenation index; oxygen supplement modalities; intubation date; IMV duration; treatment (antiviral drugs, antibiotics, glucocorticoids and vasopressor agents) or advanced therapies (intensive care unit (ICU) care, continuous renal-replacement therapy (CRRT), prone position ventilation and extracorporeal membrane oxygenation (ECMO); complications with death and other clinical outcomes. Reports of chest radiographs were obtained, and any factors associated with acute respiratory failure warranting intubation in the cohort and with death were recorded.

\section{Outcomes}

The outcomes were intubation and mechanical ventilation and in-hospital death. Based on 1-week cut-off point, we defined intubation within 7 days of ARDS diagnosis as 'early' intubation and that performed from the eighth day as 'late' intubation. The patients were then classified into three categories: 1) 'early' intubation: IMV performed within 7 days of ARDS diagnosis, 2) 'late' intubation: IMV performed from the eighth day onwards since ARDS diagnosis, and 3 ) no intubation (non-invasive respiratory support): the use of nasal cannula and facemask to inhale oxygen with no requirement of intubation during the hospital stay.

\section{Statistical analysis}

Continuous variables are expressed as medians and interquartile ranges (IQRs). Categorical variables are summarized as counts ( $n$ ) and percentages (\%). Missing data were not imputed. We used Kruskal-Wallis rank test and Fisher's exact test to compare the corresponding results among non-invasive respiratory support and early and late intubation groups. Given the incidences of all outcomes investigated exceeding $15 \%$ in which odds ratio would overstate the risk ratio, perhaps dramatically, the log-binomial regression models were used to yield risk ratios of the risk factors for intubation, late intubation and inhospital death [22]. The risk ratios (RRs) were reported if appliable. The Kaplan-Meier statistic and the survival curves were used to estimate the risk of mortality among comparisons. A two-stage procedure was performed for comparing hazard ratios among three groups. Due to the explorative nature of the study, no formal hypotheses were implemented to drive the sample size calculation, and we included the maximum number of patients who met the inclusion criteria. $P$ values less than 0.05 were considered significant except for $\mathbf{0 . 0 1 2 5}$ for two-stage procedure statistics. All analyses were performed in SPSS (version 25, IBM Corp, Armonk, NY, USA) and Stata (version 16; Stata Corp, College Station, TX, USA).

\section{Results}

Characteristics and clinical outcomes of patients with COVID-19-related ARDS 
From 26 January to 1 April 2020, 338 patients with COVID-19 confirmed by laboratory findings or otherwise clinical diagnosis were treated at the two hospitals in Hubei province of China. Of these, 70 patients developed ARDS. We included 65 of these cases with laboratory confirmation and excluded five cases. The details of enrolment are provided in Fig. 1.

The median age of the 65 included ARDS patients was 67.0 years (IQR, 56.0-78.0), with the $53.8 \%$ over 65 years and the $70.8 \%$ males (Table 1). The median body mass index (BMI) was $24.4 \mathrm{~kg} / \mathrm{m}^{2}$ (IQR, 22.426.7). More than two-thirds of patients $(n=44)$ had at least three symptoms. The most three self-reported symptoms were fever $(84.6 \%)$, cough $(70.8 \%)$, and shortness of breath $(43.1 \%)$. Vital signs evaluated at hospital admission revealed fever in 23 patients (35.4\%) and median 20.0 breaths per minute (IQR, 20.025.0). Forty-four patients had at least one comorbidity. More than two-thirds of the patients $(n=45)$ were graded as severe pneumonia by physicians. From the symptom onset to hospital admission the median number of days was 8.0 (IQR, 5.0-10.0), from hospital admission to ARDS diagnosis it was only 1.0 (IQR, 0.0-4.0), from symptom onset to ARDS diagnosis it was 11.0 (IQR, 8.0-13.0). All patients demonstrated bilateral pulmonary infiltrates. The median oxygenation index (OI) at ARDS diagnosis was 184.5 (IQR, 134.0-230.0) and 47.7\% of all patients had moderate ARDS at the beginning (OI between 199 and 100) (Table 1). Most patients received antiviral and empiric antibiotic therapies. The $78.5 \%$ of the patients took methylprednisolone (glucocorticoid) and $24.6 \%$ took vasopressors. Nearly half required ICU-level care, eight adopted prone position ventilation, and six and four received CRRT and ECMO respectively, to sustain life. The median ICU and hospital stays were 15.0 days (IQR, 10.0-20.0) and 21.0 days (IQR, 12.0-34.0). Twenty-four deaths (38.1\%) occurred, and the main cause was acute respiratory condition (62.5\%). For mild, moderate, and severe ARDS, the mortality rates were $20.0 \%, 41.9 \%$ and $85.7 \%$ respectively.

Laboratory indices tested at admission (Table 2) suggested that most patients had systemic hyperinflammation, indicated by the increased erythrocyte sedimentation rate (ESR), C-reactive protein level and procalcitonin level. Quite a few patients demonstrated reduced albumin/globulin ratio and increased total bilirubin and aspartate transaminase levels, suggesting liver injury, and elevated lactate dehydrogenase and cardiac troponin I levels, indicating heart injury. Approximately four-fifths of the patients had lymphocytopenia. Some patients were suspected of having pancreatic injury indicated by the high blood glucose level, and kidney injury based on the elevated serum creatinine level and reduced glomerular filtration rate (GFR). Next, a high international normalized ratio, prolonged prothrombin time and elevated D-dimer level were reported in several patients. Some patients demonstrated leukocytosis, neutrophilia, thrombocytopenia and reduced hemoglobin concentrations.

Comparisons among patients with non-invasive respiratory supports, early, and late intubation 
Compared with the late-intubation group, patients with early intubation had significantly shorter time from symptom onset to intubation ( $P=0.023)$, from hospital admission to intubation $(P=0.011)$, and from ARDS diagnosis to intubation $(P<0.001)$ (Fig. $2 A)$. We observed the differences on ol values $(P=$ $0.015)$ at ARDS diagnosis and proportions of ARDS classifications $(P=0.052)$ among no-intubation, early-intubation, and late-intubation groups. Patients with early intubation presented the lowest $\mathrm{OI}$ ratios (median, 133.9; IQR 98.3-183.2) and the highest proportion of moderate and severe ARDS (90\%). But patients with late intubation had a slightly higher OI ratios (median, 159.0; IQR 134.0-203.0) compared to early-intubation group. Consequently, these late intubation patients received the most aggressive therapies including glucocorticoids (100.0\%), vasopressors (50.0\%), CRRT (50.0\%), prone position ventilation (50.0\%), ECMO (50.0\%), and ICU-level care (80.0\%) in the context of the associated differences occurring among the three groups $(P=0.016, P=0.007, P<0.001, P=0.001, P<0.001, P<0.001)$. The mortality rate at discharge in the late-intubation group was $90.0 \%$ compared with $50.0 \%$ in the earlyintubation group and $23.3 \%$ in the no-intubation group $(P<0.001$, Table 3, Fig. $2 B)$. The hospital survival probability of the late-intubation group was the lowest among the three groups (Fig. $2 \mathrm{C}$ ).

Risk factors associated with intubation and hospital death

As summarized in Table 4, patients aged equal and older than 65 years (RR, 2.56; 95\% $\mathrm{Cl} 1.06-6.18, \mathrm{P}=$ 0.037), had fatigue ( $R R, 2.63 ; 95 \% \mathrm{Cl} 1.30-5.31, \mathrm{P}=0.007$ ), hypertension (RR, 2.05; 95\% $\mathrm{Cl} 1.03-4.08, \mathrm{P}=$ $0.042)$, GFR $<80 \mathrm{ml} / \mathrm{min} / 1.73 \mathrm{~m} 2(\mathrm{RR}, 2.13 ; 95 \% \mathrm{Cl} 1.02-4.48, \mathrm{P}=0.045)$ at admission, lower Ol ratio (higher values $\mathrm{RR}, 0.99 ; 95 \% \mathrm{Cl} 0.99-0.99, \mathrm{P}=0.000$ ), or Ol ratio less than 150 (RR, 2.61; $95 \% \mathrm{Cl} 1.25-5.43$, $P=0.010)$ at ARDS diagnosis were more likely to have the intubation.

Regarding hospital death, the ARDS patients on the intubation had $3.01 \mathrm{RR}(95 \% \mathrm{Cl}, 1.63-5.62, \mathrm{P}=0.000)$, and the patients receiving the late intubation had 3.18 RR $(95 \% \mathrm{Cl}, 1.98-5.12, \mathrm{P}=0.000)$. ARDS patients who were older ( $R R, 2.56 ; 95 \% \mathrm{Cl} 1.06-6.18, \mathrm{P}=0.037)$, aged equal and over 65 years (RR, 2.56; $95 \% \mathrm{Cl}$ 1.06-6.18, $\mathrm{P}=0.037$ ), had fatigue ( $R R, 2.56 ; 95 \% \mathrm{Cl}, 1.01-6.08, \mathrm{P}=0.047$ ) were more likely to die in hospital. Patients demonstrating lower Ol ratio (higher values $\mathrm{RR}, 0.99 ; 95 \% \mathrm{Cl}, 0.99-1.00, \mathrm{P}<0.001$ ), OI ratios lower than $200(\mathrm{RR}, 2.50 ; 95 \% \mathrm{Cl}, 1.07-5.83, \mathrm{P}=0.034), 150(\mathrm{RR}, 2.43 ; 95 \% \mathrm{Cl}, 1.30-4.57, \mathrm{P}=0.006$ ) or 100 (RR, $2.67 ; 95 \% \mathrm{Cl}, 1.64-4.34, \mathrm{P}<0.001)$ were associated with hospital death.

\section{Discussion}

In this study, we found that the ARDS caused by COVID-19 was not the typical ARDS defined by the Berlin criteria [20] due to longer onset time, multiple organs injuries and higher mortality in moderate and severe form. The late intubation group, but not the early intubation group, with less severity at admission, 
needed more aggressive therapies and cares, and was highly associated with in-hospital death. The predictors for intubation were not same as those for death.

The study complements the existing studies on COVID-19-related ARDS and enriches the understanding of its clinical features. The median onset time for COVID-19-related ARDS was 11.0 days (IQR, 8.0-13.0), which is inconsistent with the 1-week onset limit reported for ARDS caused by other factors [20]. The findings should alert physicians to pay more attention to the development of ARDS in COVID-19 patients with a disease course of more than a week for timely treatment. The mortality rates of moderate and severe COIVID-19-related ARDS were $41.9 \%$ and $85.7 \%$ in our study, which are much higher than $32 \%$ and $45 \%$ as reported in typical ARDS [23]. One possible explanation is silent hypoxemia as proposed by several experts, that clinical symptoms are inconsistent with the severities of hypoxemia which would result in delay treatment $[6,24]$. From the perspective of therapy, a more suitable classification of ARDS severity that can accurately identify patients requiring a timing therapy for COVID-19 has been proposed [5].

The majority of patients with ARDS in this study were men over 65 years old with hypertension and/or diabetes but not overweight. A large proportion of the patients had palpitations and chest distress at admission and later demonstrated injuries in the heart, liver, kidney, and pancreas during hospitalization. This finding is consistent with the previous findings that the new coronavirus can attack the tissues of other organs in addition to the respiratory tract [25-28]. Fundamental experiments revealed the mechanism that SARS-CoV-2 can enter the circulation by infecting the endothelium of lung tissue; the virus then directly attacks the other organs or triggers a systematic hyperinflammation response, resulting in secondary attacks to the organs [29].

We noticed that apart from antiviral therapy, many other antibiotics were administered to combat bacterial co-infections, and some patients received glucocorticoids to alleviate hyperinflammation indicated by the increasing levels of ESR, C-reactive protein and procalcitonin. We observed that patients with COVID-19-related ARDS presented with severe lymphocytopenia on admission, required aggressive therapies such as CRRT and ECMO, needed long hospital stays (median, 21.0 (IRQ, 12.0-34.0) days), but died more in hospital. All in all, updating the management strategy is highly suggested, including monitor longer to the development of COVID-19-related ARDS and re-categorize the atypical ARDS to give timing therapies.

Another important finding was that the groups of early and late intubations and the early intubation was not associated with in-hospital death. By contrast, the late-intubation group required more advanced therapies, presented a higher RR for death and trended the lowest hospital survival probabilities among the three groups. The conclusion is consistent with one study that each additional day between hospital admission and intubation was significantly associated with higher in-hospital death [15]. Because most of patients died of acute respiratory failure, the explanation coming forth that cannot be excluded was the delayed intubation. In our cohort, we noticed patients intubated late were not sicker at admission than those intubated early and their median OI ratios at ARDS diagnosis were slightly above the threshold of 
150 , where the intubation census usually suggests perform invasive mechanical ventilation if OI below 150 [16]. Given that the COVID-19-caused ARDS could present silent hypoxemia [6], where the degrees of dyspnea are inconsistent with the severities of hypoxemia, that would result in delayed intubation once sudden decompensation occurs. Many years ago, Shoemaker et al. [30] reported there is a close association between the oxygen debt accumulated over 48 hour and the chance of survival in critical patients. We noticed that even early intubation group had $50 \%$ in-hospital mortality in the cohort, as similar as the overall global data calculated recently by the systematic review [13]. Our current ventilatory approach or intubation criteria is to delay intubation if it clinically appears safe and feasible [18]. However, the findings of the cohort may suggest proactive intubation instead of salvage means for critical illness of COVID-19. This is supported by some accurate observations that many of COVID-19 patients deteriorated precipitously and that they may be more safely intubated at an earlier stage $[6,7]$. Additionally, a high work of breathing generating large swings in intrapleural pressure may result in selfinflicted lung injury and worsen the disease [7], as certainly a rationale for early intubation. However, the exact definition of the early intubation for COVID-19 critical illness is challenging. To intubate the patients early before sudden decompensation occurs while avoiding the risk of iatrogenic injury should be considered simultaneously in the face of so much high mortality.

The predictors for intubation and death were not same. The cohort found that the COVID-19 ARDS patients who had hypertension or GFR $<80 \mathrm{ml} / \mathrm{min} / 1.73 \mathrm{~m}^{2}$ were more likely to have the intubation but not death. This may suggest different development mechanism between death and intubation for COVID19 ARDS patients.

We found lower OI ratio and OI less than 150 were associated with intubation and death. OI ratio less than $\mathbf{2 0 0}$ or $\mathbf{1 0 0}$ was associated with just death. Lower OI ratios mean acute respiratory failure and 0 I less than 150 already indicates extreme respiratory failure [31]. The OI predictors for intubation or death reflecting the severity of acute lung injury as well were not reported in any study [31-34]. Our findings on Ol ratio predictors shall support the strategy that "patients with a Ol ratio less than $300 \mathrm{mmHg}$ should be prepared for intubation if there are indications, such as poor oxygenation after 2 hours of high-flow oxygen therapy or non-invasive ventilation, and OI less than 150" [16]. That requires early recognition and timing treatment of patients with worsening respiratory function during COVID-19 treatment. Additionally, we also found older ages could predict the death in COVID-19-related ARDS patients. The older age was therefore verified as the risk factor for death of critical patients as it for general COVID-19 patients [33]. The limited hospital resources and treatment strategy therefore shall focus on the elder age to reduce the death numbers from critical illness of COVID-19.

The strengths of the study are as follows: the study cohort included patients from two hospitals in two cities of Hubei province, which increases the generalisability of the findings; phenotyping of clinical characteristics was performed based on respiratory support strategies and IMV-associated groups, defined as early and late intubation according to 1-week cut-off point; reported adjustment RRs instead of crude RRs. However, the study has some limitations. First, it is a retrospective cohort conducted at two hospitals. As is known, it was hard to perform a perspective cohort when epidemic happened and 
developed everywhere. As we have sparse new COVID-19 cases now, we hope our new findings can encourage a larger cohort study or even randomly controlled trials in other regions who are experiencing the outbreak and in the urgent need to adjust the strategy. Second, the information such as respiratory function variables during the treatment course at the early and late intubations should be collected and analysed. The current cochort's findings propose the need to further explore the better timing of intubation for reducing mortality.

\section{Conclusions}

We found that the SARS-CoV-2-caused ARDS was not typical ARDS for which the management strategies should be adjusted accordingly. We studied the early- and late-intubation groups, based on 1-week cutting point, which had significant differences in the days from symptoms onset/hospital admission/ARDS diagnosis to intubation. Compared with the early intubation, the late intubation presenting less severity at admission but required more advanced treatment and had the higher risk of death. Predictors of the intubation were not same as those of in-hospital death. The findings had implications in modulating the management strategy for ARDS of COVID-19, and in supporting the early intubation strategy.

\section{Abbreviations}

\section{COVID-19}

coronavirus disease 2019; IMV:invasive mechanical ventilation; CFR:case fatality rate; Ol:oxygenation index; CRRT:continuous renal-replacement therapy; ECMO:extracorporeal membrane oxygenation; WHO:World Health Organization; ICU:intensive care unit; Pa02/Fi02:arterial partial pressure of oxygen/fraction of inspired oxygen; Sp02/Fi02:oxygen saturation by pulse oximetry/fraction of inspired oxygen; PEEP:positive end-expiratory pressure; RRs:risk ratios; IQR:interquartile range; Cl:confidence intervals; SARS-CoV-2:severe acute respiratory syndrome coronavirus 2; BMl:body mass index; ESR:erythrocyte sedimentation rate; GFR:glomerular filtration rate; WBC:white blood cell; TBIL:total bilirubin; AST:aspartate transaminase; ALT:alanine aminotransaminase; LDH:lactate dehydrogenase; GFR:glomerular filtration rate; PT:prothrombin time; INR:international normalized ratio; ESR:erythrocyte sedimentation rate;SBP:systolic blood pressure; DBP:diastolic blood pressure; MAP:mean arterial pressure

\section{Declarations}

\section{Ethics approval and consent to participate}

This manuscript adheres to the applicable CONSORT guidelines. The study was approved by the Chinese Ethics Committee of Registering Clinical Trials (ChiECRCT20200113), and the requirement for informed consent was waived because of the retrospective study design and given the context of ongoing infectious diseases. 
Not applicable

\section{Availability of data and materials}

After publication, the data will be made available to others on reasonable requests to the corresponding author. A proposal with detailed description of study objectives and statistical analysis plan will be needed for evaluation of the reasonability of requests. Additional materials might also be required during the process of evaluation. Deidentified participant data will be provided after approval from the corresponding author and Wuhan and Hong Hu Hospitals.

\section{Competing interests}

The authors declare that they have no competing interests.

\section{Funding}

This work was supported by the National Natural Science Foundation of China $(81902009,81730058$, 81671955), Beijing, China.

\section{Authors' contributions}

JLM: This author helped with study design, data collection, write the manuscript and edit for critical content.

MZQ: This author helped with data collection and edit for critical content.

HMS: This author helped with data collection and edit for critical content.

BSG: This author helped with data analysis and edit for critical content.

DSQ: This author helped with data collection and data analysis.

FZ: This author helped with data collection and data analysis.

HY: This author helped with data collection and data analysis.

JN: This author helped with data collection and edit for critical content.

XuD Liu: This author helped with data analysis and edit for critical content

XiaoDL: This author helped with data analysis, interpretation, and edit for critical content

WFY: This author helped with data interpretation and edit for critical content

BXL: This author helped with data collection and edit for critical content. 
XYS: This author helped with data collection and edit for critical content.

ShYL: This author helped with edit for critical content.

YHD: This author helped with data collection and edit for critical content.

KXL: This author helped with study design, data interpretation, and edit for critical content.

\section{Acknowledgements}

We thank all patients involved in the study, residents, and nurses who helped with data collection. We appreciate Prof. Hao Liu for his advice on manuscript improvement. We would like to thank Dr. Hua Jiang, the frontline expert of COVID-19 management, for her advice on the data interpretation. We are very thankful for Secretary Ms. Jing-Xin Xiao for her assistance in conducting the research.

\section{Author details.}

Department of Anesthesiology, Nanjing Drum Tower Hospital, Nanjing University Medical School Affiliated, Nanjing, Jiangsu, China (JL Mu MD)

2. Department of Anesthesiology, Nanfang Hospital, Southern Medical University, Guangzhou, Guangdong, China (JL Mu MD, SD Qiu MD, F Zhang MD, H Yang MMed, Ye-Hong Dong, MD, Prof. KX Liu MD, PhD)

3. Department of Anesthesiology, General Hospital of Central Theatre Command of PLA, Wuhan, Hubei, China (MZ Qin MD, BX Li MD, XY Song MD)

4. Department of Cardiovascular Surgery, Nanfang Hospital, Southern Medical University, Guangzhou, Guangdong, China (HM Sun BSN, ShY Lin BSN)

5. State Key Laboratory of Pharmaceutical Biotechnology, Department of Sports Medicine and Adult Reconstructive Surgery, Nanjing Drum Tower Hospital, the Affiliated Hospital of Nanjing University Medical School, Nanjing, Jiangsu, China (Prof. BS Guo, PhD)

6. Department of Infection, The People's Hospital of Honghu, Jingzhou, Hubei, China (J Nie BSN)

7. School of Public Health, Sun Yat-sen University, Guangzhou, Guangdong, China (Prof. XuD Liu PhD)

8. School of Nursing, The Hong Kong Polytechnic University, Hong Kong Special Administrative Region of China (Dr. WF Yeung PhD)

9. Department of Anesthesia and Intensive Care, Prince of Wales Hospital, The Chinese University of Hong Kong, Hong Kong Special Administrative Region of China (Prof. XiaoD Liu PhD)

\section{References}

1. WHO. World Health Organization. https://covid19.who.int/. Accessed Feb 272021. 
2. Baksh M, Ravat V, Zaidi A, Patel RS. A Systematic Review of Cases of Acute Respiratory Distress Syndrome in the Coronavirus Disease 2019 Pandemic. Cureus. 2020;12(5):e8188.

3. Wu C, Chen X, Cai Y, Xia Ja, Zhou X, Xu S, et al. Risk Factors Associated With Acute Respiratory Distress Syndrome and Death in Patients With Coronavirus Disease 2019 Pneumonia in Wuhan, China. JAMA Internal Medicine. 2020;180:1-11.

4. Fan E, Beitler JR, Brochard L, Calfee CS, Ferguson ND, Slutsky AS, et al. COVID-19-associated acute respiratory distress syndrome: is a different approach to management warranted? The Lancet. Respiratory medicine. 2020;8(8):816-21.

5. Li X. and X. Ma Acute respiratory failure in COVID-19: is it "typical" ARDS? Critical care (London, England). 2020.24(1):198.

6. Rola P, Farkas J, Spiegel R, Kyle-Sidell C, Weingart S, Duggan L, et al. Rethinking the early intubation paradigm of COVID-19: time to change gears? Clinical and experimental emergency medicine. 2020.7(2):78-80.

7. Marini JJ. and L. Gattinoni Management of COVID-19 Respiratory Distress. JAMA. 2020;323(22):2329-30.

8. Guan W, Ni Z, Hu Y, Liang W, Ou C, He J, et al. Clinical Characteristics of Coronavirus Disease 2019 in China. N Engl J Med. 2020;382(18):1708-20.

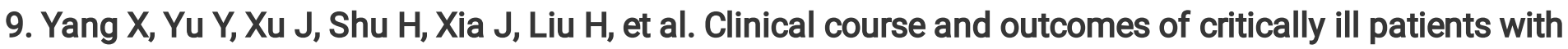
SARS-CoV-2 pneumonia in Wuhan, China: a single-centered, retrospective, observational study. Lancet Respir Med. 2020;8(5):475-81.

10. Cummings MJ, Baldwin MR, Abrams D, Jacobson SD, Meyer BJ, Balough EM, et al. Epidemiology, clinical course, and outcomes of critically ill adults with COVID-19 in New York City: a prospective cohort study. Lancet. 2020;395(10239):1763-70.

11. Grasselli G, Greco M, Zanella A, Albano G, Antonelli M, Bellani G, et al. Risk Factors Associated With Mortality Among Patients With COVID-19 in Intensive Care Units in Lombardy, Italy. JAMA Internal Medicine. 2020;180(10):1345-55.

12. Bhatraju PK, Ghassemieh BJ, Nichols M, Kim R, Jerome KR, Nalla AK, et al. Covid-19 in Critically III Patients in the Seattle Region - Case Series. N Engl J Med. 2020;382(21):2012-22.

13. Lim ZJ, Subramaniam A, Reddy MP, Blecher G, Kadam U, Afroz A, et al. Case Fatality Rates for COVID-19 Patients Requiring Invasive Mechanical Ventilation: A Meta-analysis. Am J Respir Crit Care Med. 2021;203(1):54-66.

14. de Simone G, Mancusi C. COVID-19: Timing is Important. European journal of internal medicine. 2020;77:134-5.

15. Hyman JB, Leibner ES, Tandon P, Egorova NN, Bassily-Marcus A, Kohli-Seth R, et al. Timing of Intubation and In-Hospital Mortality in Patients With Coronavirus Disease 2019. Critical Care Explorations. 2020;2(10):e0254.

16. Meng L, Qiu H, Wan L, Ai Y, Xue Z, Guo Q, et al. Intubation and Ventilation amid the COVID-19 Outbreak: Wuhan's Experience. Anesthesiology: The Journal of the American Society of 
Anesthesiologists. 2020;132(6):1317-32.

17. Gattinoni L, Chiumello D, Caironi P, Busana M, Romitti F, Brazzi L, et al. COVID-19 pneumonia: different respiratory treatments for different phenotypes? Intensive Care Med. 2020;46(6):1099-102.

18. Xiao N, Cooper JG, Godbe JM, Bechel MA, Scott MB, Nguyen E, et al. Chest radiograph at admission predicts early intubation among inpatient COVID-19 patients. European Radiology. 2020:1-8.

19. Novel coronavirus - China. http://www.who.int/csr/don/12-january-2020-novel-coronaviruschina/en/. Accessed Jan 192020.

20. The ARDS Definition Task Force. Acute Respiratory Distress Syndrome: The Berlin Definition. JAMA. 2012;307(23):2526-33.

21. ISARIC WHO SARI Case Record Form. https://isaric.tghn.org/. Accessed May 52020.

22. McNutt LA, Wu C, Xue X. and J.P. Hafner Estimating the relative risk in cohort studies and clinical trials of common outcomes. Am J Epidemiol. 2003;157(10):940-3.

23. Fanelli V, Vlachou A, Ghannadian S, Simonetti U, Slutsky AS. and H. Zhang Acute respiratory distress syndrome: new definition, current and future therapeutic options. Journal of thoracic disease. 2013;5(3):326-34.

24. Nickson C, Young IJ. P. "Silent hypoxaemia" and COVID-19 intubation. https://litfl.com/silenthypoxaemia-and-covid-19-intubation/. Accessed Feb 52021.

25. Liu F, Long X, Zou W, Fang M, Wu W, Li W, et al. Highly ACE2 Expression in Pancreas May Cause Pancreas Damage After SARS-CoV-2 Infection. Clin Gastroenterol Hepatol. 2020;18(9):2128-30.e2.

26. Fan Z, Chen L, Li J, Tian C, Zhang Y, Huang S, et al. Clinical Features of COVID-19 Related Liver Damage. Clin Gastroenterol Hepatol. 2020;18(7):1561-6.

27. Diao B, Wang C, Wang R, Feng Z, Tan Y, Wang H, et al. Human Kidney is a Target for Novel Severe Acute Respiratory Syndrome Coronavirus 2 (SARS-CoV-2) Infection. medRxiv. Preprint posted online 10 April 2020.

28. Bonow RO, Fonarow GC, O'Gara PT. and C.W. Yancy Association of Coronavirus Disease 2019 (COVID-19) With Myocardial Injury and Mortality [Editorial]. JAMA Cardiology. 2020;5(7):751-3.

29. Takayama K. In Vitro and Animal Models for SARS-CoV-2 research. Trends Pharmacol Sci. 2020;41(8):513-17.

30. Shoemaker WC, Appel PL. and H.B. Kram Role of oxygen debt in the development of organ failure sepsis, and death in high-risk surgical patients. Chest. 1992;102(1):208-15.

31. Pinson R, Revisiting. respiratory failure. https://acphospitalist.org/archives/2013/11/coding.htm. Accessed Feb 52021.

32. Sarkar M, Niranjan N. and P.K. Banyal Mechanisms of hypoxemia. Lung India: official organ of Indian Chest Society. 2017;34(1):47-60.

33. Chen L, Yu J, He W, Chen L, Yuan G, Dong F, et al. Risk factors for death in 1859 subjects with COVID19. Leukemia. 2020;34(8):2173-83. 
34. Williamson EJ, Walker AJ, Bhaskaran $\mathrm{K}$, Bacon S, Bates $\mathrm{C}$, Morton $\mathrm{CE}$, et al. Factors associated with COVID-19-related death using OpenSAFELY. Nature. 2020;584(7821):430-6.

\section{Tables}

Table 1. Demographics, characteristics, and outcomes of COVID-19 patients developing ARDS 
Study variables

No. of patients

Age

median, IQR

$\geq 65$ years

Male sex

BMI*

median, IQR

$\geq 25$

Smoke history

Alcohol used

Days from symptoms onset to hospital admission (median, IQR)

Initial symptoms

Fever

Cough

Short of breath

Fatigue

Expectoration

Palpitation and chest distress

Number of complaints $\geq 3$

Vital signs on admission

Fever on admission $\left(\geq 37.2^{\circ} \mathrm{C}\right)$

Breath rate (median, IQR)

Heart rate (median, IQR)

SBP (median, IQR)

DBP (median, IQR)

MAP (median, IQR)
Value, no./total no. (\%) or median (IQR)

65

$67.0(56.0-78.0)$

$35 / 65$ (53.8\%)

$46 / 65$ (70.8\%)

24.4 (22.4-26.7)

$20 / 65$ (30.8\%)

$7 / 65$ (10.8\%)

9/65 (13.8\%)

$8.0(5.0-10.0)$

$55 / 65$ (84.6\%)

$46 / 65$ (70.8\%)

28/65 (43.1\%)

20/65 (30.8\%)

$17 / 65$ (26.2\%)

$17 / 65(26.2 \%)$

$44 / 65$ (67.7\%)

23/65 (35.4\%)

$20.0(20.0-25.0)$

$90.0(79.0-98.0)$

$132.0(122.0-142.0)$

$78.0(71.0-88.0)$

$96.7(89.0-105.0)$

Severity of pneumonia graded by physicians 
Mild

Severe

Critical

Comorbidities

Hypertension

Diabetes

Cardiovascular disease

Brain disease

Chronic lung disease

Number of coexisting disorders $\geq 1$

Days from hospital admission to ARDS diagnosis (median, IQR)

Days from symptoms onset to ARDS diagnosis (median, IQR)

Chest imaging, bilateral infiltrate

Oxygenation Index on ARDS Diagnosis

median (IQR)

$300-200$

$199-100$

$<100$

Treatments

Antiviral therapy

Intravenous antibiotic

Glucocorticoid (methylprednisolone)

Vasopressor

Admission to ICU

Prone positioning

Continuous renal-replacement therapy

ECMO

Outcomes
$2 / 65(3.1 \%)$

$18 / 65$ (27.7\%)

$45 / 65$ (69.2\%)

$20 / 65$ (30.8\%)

$10 / 65$ (15.4\%)

$8 / 65(12.3 \%)$

$6 / 65(9.2 \%)$

$2 / 65(3.1 \%)$

$44 / 65(67.7 \%)$

$1.0(0.0-4.0)$

$11.0(8.0-13.0)$

$65 / 65(100 \%)$

184.5 (134.0-230.0)

27/65 (41.5\%)

$31 / 65$ (47.7\%)

$7 / 65(10.8 \%)$

63/65 (96.9\%)

$60 / 65$ (92.3\%)

$51 / 65$ (78.5\%)

$16 / 65$ (24.6\%)

29/65 (44.6\%)

$8 / 65$ (12.3\%)

$6 / 65(9.2 \%)$

$4 / 65(6.2 \%)$ 


\begin{tabular}{|ll|}
\hline Failure of noninvasive respiratory support* & $20 / 65(31.7 \%)$ \\
\hline Median ICU stay (day, IQR) + & $15.0(10.0-20.0)$ \\
\hline Median Hospital stay (day, IQR) & $21.0(12.0-34.0)$ \\
\hline Death in hospital & $24 / 65(36.9 \%)$ \\
\hline Death in OI $\geq 200$ & $5 / 25(20.0 \%)$ \\
\hline Death in $200>$ OI $\geq 100$ & $13 / 31(41.9 \%)$ \\
\hline Death in OI < 100 & $6 / 7(85.7 \%)$ \\
\hline Death due to acute respiratory failure & $15 / 24(62.5 \%)$ \\
\hline
\end{tabular}

* All patients first were on noninvasive oxygen support when admitted at hospital.

† Data summarized from 29 patients admitted into ICU.

Abbreviations: Ol: Oxygenation Index, ECMO: Extracorporeal membrane oxygenation, MODS: Multiple Organ Dysfunction Syndrome.

Table 2. Initial laboratory indices of COVID-19 patients developing ARDS 


\begin{tabular}{|c|c|c|c|}
\hline $\begin{array}{l}\text { Laboratory indices on } \\
\text { admission }\end{array}$ & $\begin{array}{l}\text { Reference } \\
\text { values }\end{array}$ & $\begin{array}{l}\text { Value, median } \\
\text { (IQR) }\end{array}$ & $\begin{array}{l}\text { No. of patients with value deviation from } \\
\text { reference (\%) }\end{array}$ \\
\hline \multicolumn{4}{|l|}{ Hematologic } \\
\hline $\begin{array}{l}\text { Lymphocyte count } \\
\text { /mm3 }\end{array}$ & $1000-4800$ & $\begin{array}{l}670(430- \\
1040)\end{array}$ & $47 / 65(72.3 \%)^{b}$ \\
\hline WBC count /mm3 & $4000-10000$ & $\begin{array}{l}7340(4700- \\
10700)\end{array}$ & $19 / 65(29.2 \%)^{a}$ \\
\hline Neutrophil count /mm3 & $2500-8000$ & $\begin{array}{l}6320(3440- \\
9150)\end{array}$ & $21 / 65(32.3 \%)^{a}$ \\
\hline Platelet count /mm3 & $150-450$ & $199(146-236)$ & $16 / 65(24.6 \%)^{\mathrm{b}}$ \\
\hline Hemoglobin, g/dL & $12-17.5$ & $\begin{array}{l}13.1(11.6- \\
14.0)\end{array}$ & $20 / 65(30.8 \%)^{b}$ \\
\hline \multicolumn{4}{|l|}{ Biochemical } \\
\hline TBIL, $\mu \mathrm{mol} / \mathrm{L}$ & $3.4-17.1$ & $\begin{array}{l}13.0(10.1- \\
18.3)\end{array}$ & $48 / 65(73.8 \%)^{a}$ \\
\hline AST, U/L & $10-40$ & $\begin{array}{l}39.0(25.0- \\
50.0)\end{array}$ & $27 / 65(41.5 \%)^{a}$ \\
\hline ALT, U/L & $7-56$ & $\begin{array}{l}27.0(20.0- \\
35.0)\end{array}$ & $5 / 65(7.7 \%)^{a}$ \\
\hline Total protein, $\mathbf{g} / \mathrm{L}$ & $60-83$ & $\begin{array}{l}64.0(60.7- \\
68.3)^{(1)}\end{array}$ & $15 / 65(23.1 \%)^{b}$ \\
\hline Albumin, $\mathrm{g} / \mathrm{L}$ & $32-45$ & $\begin{array}{l}34.1(31.2- \\
37.5)\end{array}$ & $20 / 65(30.8 \%)^{b}$ \\
\hline Albumin/Globulin & $1.5-2.5$ & $\begin{array}{l}1.13(0.95- \\
1.39)\end{array}$ & $55 / 65(84.6 \%)^{\mathrm{b}}$ \\
\hline LDH, unit/L & $140-280$ & $\begin{array}{l}371.5(227.0- \\
519.0)\end{array}$ & $40 / 65(61.5 \%)^{a}$ \\
\hline cTnl, ng/mL & $0-0.04$ & $\begin{array}{l}0.02(0.01- \\
0.04)\end{array}$ & $15 / 65(23.1 \%)^{a}$ \\
\hline Glucose, mmol/L & $3.9-7.1$ & $7.9(6.0-10.5)$ & $37 / 65(56.9 \%)^{a}$ \\
\hline Creatinine, $\mu \mathrm{mol} / \mathrm{L}$ & $57-110$ & $\begin{array}{l}74.2(59.0- \\
93.0)\end{array}$ & $12 / 65(18.5 \%)^{a}$ \\
\hline GFR, $\mathrm{ml} / \mathrm{min} / 1.73 \mathrm{~m}^{2}$ & $80-125$ & $\begin{array}{l}89.0(62.0- \\
104.0)\end{array}$ & $27 / 65(41.5 \%)^{b}$ \\
\hline Potassium, mmol/L* & $3.5-5.0$ & $3.9(3.7-4.3)$ & $11 / 59(18.6 \%)^{b}$ \\
\hline Sodium, mmol/L* & $135-145$ & $\begin{array}{l}137.8(134.8- \\
139.3)\end{array}$ & $15 / 59(25.4 \%)^{b}$ \\
\hline
\end{tabular}




\begin{tabular}{|c|c|c|c|}
\hline D-Dimer, mg/L & $0.0-0.5$ & $0.6(0.2-2.4)$ & $34 / 65(52.3 \%)^{a}$ \\
\hline PT, s & $10.5-13.5$ & $\begin{array}{l}13.1(11.9- \\
14.7)\end{array}$ & $27 / 65(41.5 \%)^{a}$ \\
\hline INR & $\leq 1.1$ & $1.1(1.1-1.2)$ & $38 / 65(58.5 \%)^{a}$ \\
\hline \multicolumn{4}{|l|}{ Inflammation related } \\
\hline ESR, mm/h† & $0-20$ & $\begin{array}{l}62.5(31.0- \\
81.0)\end{array}$ & $52 / 58(89.7 \%)^{a}$ \\
\hline $\begin{array}{l}\text { C-Reactive Protein, } \\
\mathrm{mg} / \mathrm{L}\end{array}$ & $5-10$ & $\begin{array}{l}59.1(20.1- \\
108.6)\end{array}$ & $57 / 65(87.7 \%)^{a}$ \\
\hline Procalcitonin, $\mu \mathrm{g} / \mathrm{L}$ & $\leq 0.10$ & $\begin{array}{l}0.12(0.05- \\
0.38)\end{array}$ & $35 / 65(53.8 \%)^{a}$ \\
\hline
\end{tabular}
a. Above the reference limit
b. Below the reference limit

* Data regarding the Potassium and Sodium were missing for 6 patients respectively (9.2\%).

† Data regarding the ESR were missing for 7 patients (10.8\%).

Abbreviations: WBC: White Blood Cell; TBIL: Total bilirubin, AST: aspartate transaminase, ALT: alanine aminotransaminase, LDH: lactate dehydrogenase, GFR: glomerular filtration rate, PT: prothrombin time, INR: international normalized ratio, ESR: Erythrocyte Sedimentation Rate.

Table 3. Consequences among noninvasive respiratory support and early and late intubation groups in COVID-19-related ARDS patients 


\begin{tabular}{|lllll|}
\hline $\begin{array}{l}\text { Oxygenation Index on ARDS Diagnosis, } \\
\text { median (IQR) }\end{array}$ & $200.0(158.0-$ & $133.9(98.3-$ & $159.0(134.0-$ & 0.015 \\
\hline $300-200$ & $247.5)$ & $183.2)$ & $203.0)$ & \\
\hline $199-100$ & $19 / 45(42.2 \%)$ & $6 / 10(60.0 \%)$ & $6 / 10(60.0 \%)$ & \\
\hline$<100$ & $3 / 45(6.7 \%)$ & $3 / 10(30.0 \%)$ & $1 / 10(10.0 \%)$ & 0.052 \\
\hline Systemic glucocorticoids & $31 / 45(68.9 \%)$ & $10 / 10$ & $10 / 10(100 \%)$ & 0.016 \\
\hline Vasopressor & $6 / 45(13.3 \%)$ & $5 / 10(50.0 \%)$ & $5 / 10(50.0 \%)$ & 0.007 \\
\hline $\begin{array}{l}\text { Use of continuous renal-replacement } \\
\text { therapy }\end{array}$ & $0 / 45(0.0 \%)$ & $1 / 10(10.0 \%)$ & $5 / 10(50.0 \%)$ & 0.000 \\
\hline Prone positioning & $0 / 45(0.0 \%)$ & $3 / 10(30.0 \%)$ & $5 / 10(50.0 \%)$ & 0.000 \\
\hline ECMO & $0 / 45(0.0 \%)$ & $1 / 10(10.0 \%)$ & $5 / 10(50.0 \%)$ & 0.000 \\
\hline Admission to ICU & $14 / 45(31.1 \%)$ & $7 / 10(70.0 \%)$ & $8 / 10(80.0 \%)$ & 0.005 \\
\hline Death in the hospital & $10 / 45(23.3 \%)$ & $5 / 10(50.0 \%)$ & $9 / 10(90.0 \%)$ & 0.000 \\
\hline
\end{tabular}

* Respiratory support strategies include nasal cannula inhalation, high-flow nasal cannula therapy, and noninvasive ventilation.

† Patients of hospital admission temperature $\geq 37.2^{\circ} \mathrm{C}$.

‡ Fisher's exact test used for analysing percentage data.

Abbreviations: SBP: systolic blood pressure, DBP: diastolic blood pressure, MAP: Mean Arterial Pressure, GRF: glomerular filtration rate ECMO: Extracorporeal membrane oxygenation; NA: not applicable.

Table 4. Log-binominal regression of factors associated with intubation and death 


\begin{tabular}{|c|c|c|c|c|}
\hline \multirow[t]{2}{*}{ Variables } & \multicolumn{2}{|l|}{$\begin{array}{l}\text { Intubation } \\
(n=20)\end{array}$} & \multicolumn{2}{|l|}{$\begin{array}{l}\text { Death } \\
(n=24)\end{array}$} \\
\hline & $\mathrm{RR}^{*}$ & $P$ value & $\mathrm{RR}^{*}$ & $P$ value \\
\hline \multicolumn{5}{|l|}{ Intubation Ventilation } \\
\hline Intubation & NA & NA & $3.01(1.63-5.62)$ & 0.000 \\
\hline Early intubation & NA & NA & $1.39(0.68-2.86)$ & 0.363 \\
\hline Late Intubation & NA & NA & $3.18(1.98-5.12)$ & 0.000 \\
\hline \multicolumn{5}{|l|}{ Factors } \\
\hline Age, years & $1.02(0.99-1.04)$ & 0.159 & $1.04(1.02-1.05)$ & 0.000 \\
\hline Age $\geq 65$ years & $2.56(1.06-6.18)$ & 0.037 & $3.24(1.38-7.59)$ & 0.007 \\
\hline Fatigue complaint & $2.63(1.30-5.31)$ & 0.007 & $2.15(1.18-3.91)$ & 0.012 \\
\hline Hypertension & $2.05(1.03-4.08)$ & 0.042 & $1.25(0.65-2.39)$ & 0.501 \\
\hline GFR $<80 \mathrm{ml} / \mathrm{min} / 1.73 \mathrm{~m}^{2}$ & $2.13(1.02-4.48)$ & 0.045 & $1.20(0.64-2.25)$ & 0.561 \\
\hline \multicolumn{5}{|l|}{ Oxygenation Index } \\
\hline Values & $0.99(0.99-0.99)$ & 0.000 & $0.99(0.99-1.00)$ & 0.000 \\
\hline$<200$ & $2.63(1.00-6.96)$ & 0.051 & $2.50(1.07-5.83)$ & 0.034 \\
\hline$<150$ & $2.61(1.25-5.43)$ & 0.010 & $2.43(1.30-4.57)$ & 0.006 \\
\hline$<100$ & $2.00(0.93-4.29)$ & 0.075 & $2.67(1.64-4.34)$ & 0.000 \\
\hline
\end{tabular}

*All RRs adjusted for Age $\geq 65$ years, except for age and Age $\geq 65$ years adjusted for Oxygenation Index.

† Robust Poisson regression substituting for binomial regression due to fail in converge. Abbreviations: RR; risk ratio; GFR: glomerular filtration rate

\section{Figures}




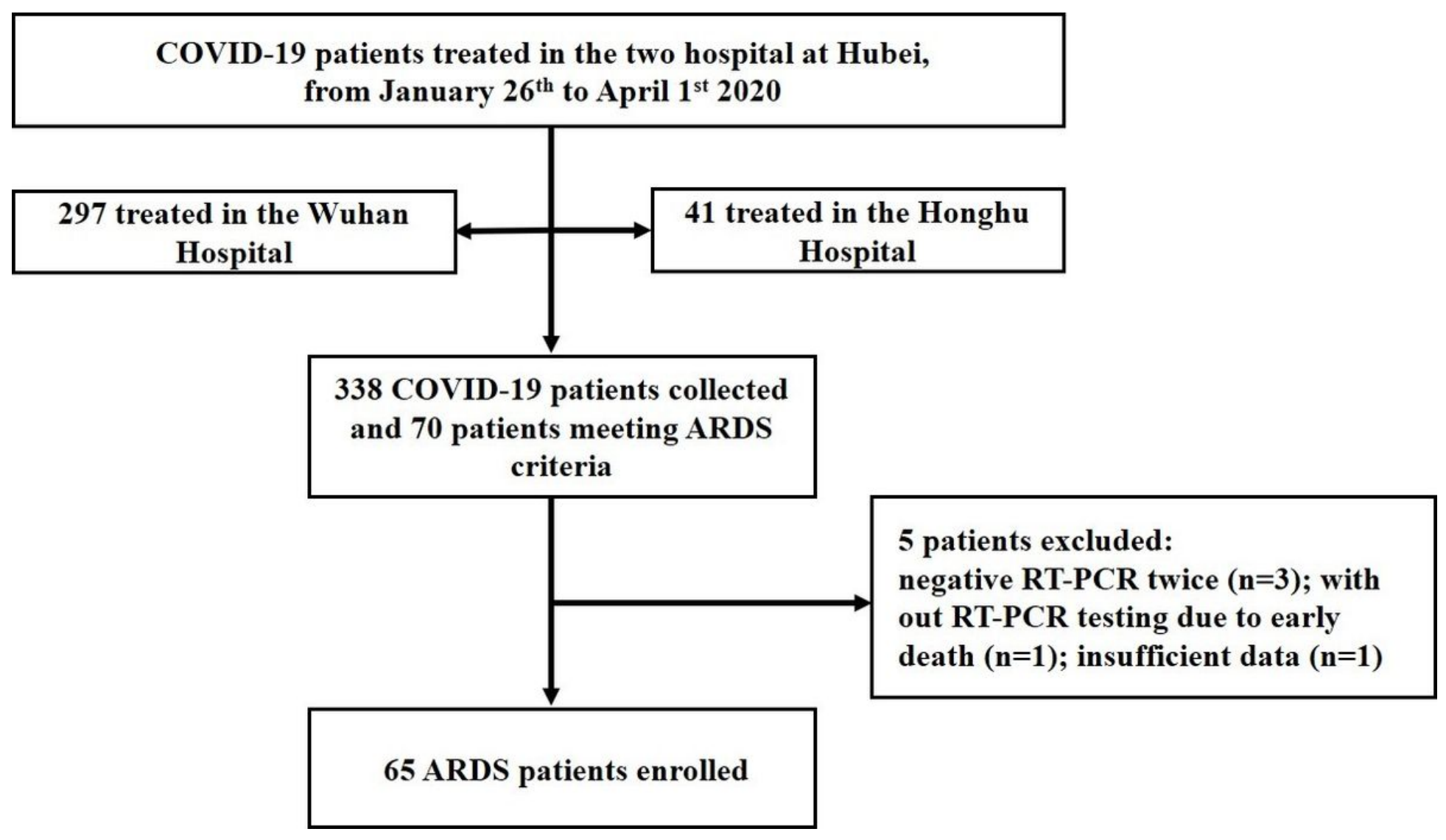

Figure 1

Enrolment of COVID-19-related ARDS patients at Wuhan and Honghu hospitals 


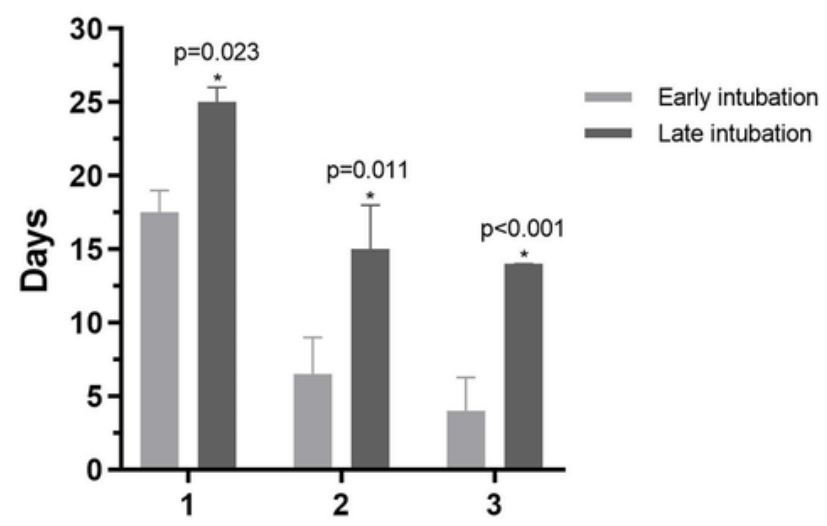

Median (IQR) days from onset of symptoms to (1) hospital admission. (2) ARDS diaqnosis, and (3) intubation

B

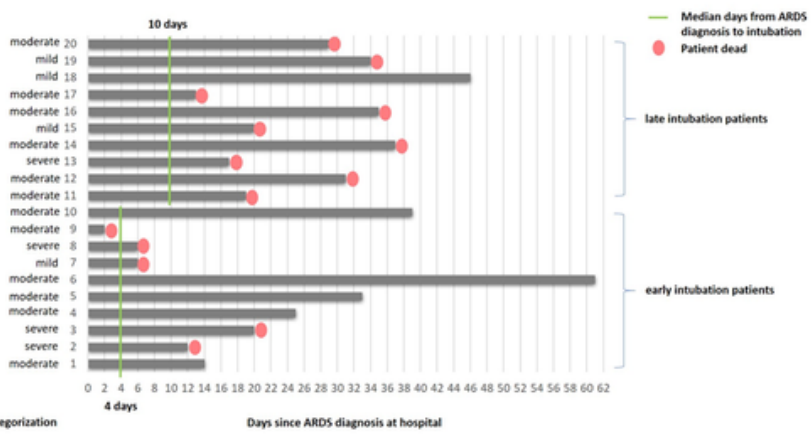

c

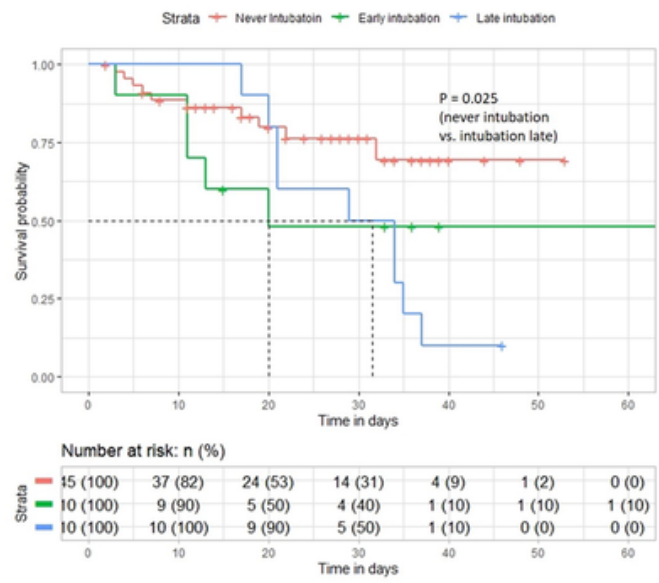

Figure 2

Information for intubation group classifications and the outcomes. A. Median (IQR) days from onset of symptoms to hospital admission, ARDS diagnosis, and intubation; B. 20 deaths and their intubation time; C. Hospital survival probability of never-, early-, and late-intubation groups. * A statistical difference based on $\mathrm{P}<0.05$. 